\title{
Missing Journals
}

\section{APPEAL REPEATED}

Below is a list of volumes of physics journals missing from science and institute libraries in Czechoslovakia [CS], Hungary $[\mathrm{H}]$, Poland [PL], and Romania [R]. The data were obtained in the EPS survey for the Library Aid programme carried out by E.W.A. Lingeman.

It is hoped that a similiar survey will by carried out shortly for the remaining east and central European countries. Meanwhile, some of these countries have sent lists of missing journals to E.W.A. Lingeman ( $\%$ EPS Budapest); others are invited to do the same.

A total of 161 volumes were donated following publication of the first list last July [EN 22 (1991) 144]. These volumes have been removed from the revised list given below.

If you wish to donate copies of any of the missing volumes please contact the EPS Secretariat, Budapest, giving details of the volume (volumes) available. You will then be sent the address of the library to which it (they) should be forwarded.

Acta Crystal. (1981-91) [CS], 42 (1986) [PL] Acta Crystallogr. B 38 (1982) [CS]

Acta Physica Austriaca 44-55 (1976-83) [CS], (1982-90) [PL]

Adv. Phys. 1-30 (1952-90) [PL], 23 (1974) [R], 30 (1981)[R], 38-39 (1989-91) [H]

Amer. J. Phys. 35 (1967) [PL], 50 (1982) [CS], 52-53 (1984-5) [PL], 56-59 (1988-91) [CS]. 56 (1988), 58 (1990) [PL]

Ann. Geophysique (1977) [CS]

Ann. Institut Henri Poincaré (1982-90) [PL]

Ann. Phys. (N.Y.) (1982), (1989-91) [H], 138-144 (1982) [CS], 142 (1982) [PL], (1983-91) [CS], 166 (1986), 188 (1988), 197-204 [PL]

Ann. Rev. Nuclear Sci. 30 (1980) [PL]

Appl. Optics (1981-90) [CS], 21 (1982), (1985-8) [PL], 26-29 (1987-90) [CS]

Appl. Phys. (1975) [CS], (1982-90) [PL]

Appl. Phys. A 27-28 (1982), 45 (1988), 50-51 (1990) [PL]

Appl. Phys. B 27-28 (1982), 39 (1986), 45 (1988), 50-51 (1990) [PL]

Appl. Phys. Lett. 30-35 (1977-8)

[CS], 36 (1980), 38-40 (1981-2), 43 (1983), 46-47 (1985) [PL], 46-57 (1985-90) [CS], 52-55 (1988-90) [PL], 56-57 (1990) [CS] Appl. Surf. Sci. (1982-6) [PL]

Astronom. Nach. 277, 278, 285, 308 [CS]

Astronom. \& Astrophys. Suppl. 48, 51, 53, 60, 86 [CS]

Astronom. \& Astrophys. 48, 60, 65, 67 [CS

Astrophys. J. (1988-91), 219, 231, 312, 315 317 [CS]

Astrophys. J. Lett. 313, 318, 319 [CS]

Astrophys. J. Suppl. 1, 2, 7, 8, 57, 65, 69 [CS] Astrophys. Lett. 15 (1989) [CS]

J. Chem. Phys. 82-85 (1985-66) [PL]

J. Phys. Chem. Solids $42-45$ (1981-89) [PL]

J. Phys.: Cond. Matter 11 (1978), 13-17 (1980-4) [PL]

J. Plasma Phys. 25 (1981), 29 (1983), 33-34 (1985), 37-38 (1987) [PL], 39-45 (1988-91) [CS]

J. Raman Spectros. 12-21 (1982-90) [CS]

J. Rheology 32-33 (1988-9) [CS]

J. Stat. Phys. 1-6 (1971-2), 17 (1977), 23-25 (1980-1), 29 (1982) [PL], 27-37 (1982-4)

[CS], 42 (1986) [PL]

J. Superconductivity 1 (1988) [CS]

J. Vac. Sci. \& Tech. 18 (1981), (1983-90), (1989-91) $[\mathrm{H}]$

J. X-Ray Sci. \& Tech. 1 (1989) [PL]

J. Electrochem. Soc. (1990) [PL]

J. Optical Soc. of Amer. A \& B (1982-4) [CS]

J. Phys. Soc. Japan 51-59 (1982-90) [PL]

Laser \& Particle Beams 1 (1983) [CS]
Lett. Nuovo Cim. (1982-91) [H], (1986-90) [PL] Lett. Math. Phys. 6-20 (1982-90) [CS]

Mater. Res. Bull. 17 (1982) [CS]

Mater. Sci. \& Engng. $32-41$ (1978-9), 52-56 (1982) [CS]

Math. Systems Theory 15 (1982) [PL]

Mercury 4, 5, 6, 7, 8 [CS]

Modern Phys. Lett. A 1-2 (1986-7) [CS],

1 (1986) [PL]

Modern Phys. Lett. B 1 (1987) [CS],

1-3 (1987-9) [PL]

Molecular Crystals \& Liquid Crystals 74 (1982), 154-190 (1988-90) [CS]

Mol. Phys. 14-22 (1967-71) [PL], 42 (1981) [R], (1981-90) [PL]

Monthly Notices Royal Astronom. Soc. (1988 91) [CS], 125, 129, 142, 148, 156, 160-169, $170-173$ [CS]

Nature (1981-90) [CS], 331-337 (1988) [PL]

Nuclear Data A 4 (1968) [PL]

Nuclear Data Sheets 1-8 (1969-72), 32 (1981), 40 (1983), 44 (1985), $45-46$ (1985), 59-61 (1990) [PL]

Nucl. Instrum. \& Methods 107-108 (1973), 190 (1981), 196 (1982), 203 (1982), 215 (1983), 230-233 (1985), 285 (1989) [PL]

Nucl. Instrum. \& Methods A 252-253 (1986-7) [PL]

Nucl. Instrum. \& Methods B 9 (1985) [PL Nuclear Phys. A (1974-90) [CS], 340 (1980), 360 (1981), 403 (1983), 421 (1984) [PL]

Nuclear Phys. B (1982-90) [CS], 216 (1983), 298 (1988) [PL]

Nuclear Phys. B; Proc. Suppl. (1987-9) [PL]

Nuclear Phys. 84, 86, 101 [CS]

Observatory 72-76, 80, 95-101, 105 [CS]

Optica Acta 1-15 (1954-68), 28 (1981) [PL]

Optica Acta: J. Modern Optics 36-37

(1989-90) [CS]

Optics Commun. 1990 [PL]

Optics Lett. (1985) [CS]

Optik 63-65 (1982-3) [PL]

Philips J. Research 38 (1983) [PL]

Phil. Mag. A/B 43 (1981) [R], 43-52 (1981-90) [PL]

Physica Scripta 26 (1982) [PL, R], 35 (1987) [PL]

Phys. Abs. (1982-90) [CS], 85 (1982), 88-92 (1985-90) [PL]

Phys. \& Fluids A (1982-90) [CS]

Phys. \& Fluids $B$ (1982-90) [CS]

Phys. Fluids 24 (1981) [PL]

Phys. Lett. (1979-90) [CS]

Phys. Lett. A (1979-90) [CS], 75 (1980), 88 (1982), 90 (1982), 120 (1987), 123 (1987), 127 (1988) [PL]

Phys. Lett. B (1987-90) [CS], 107-8 (1981-2)،
111 (1982), 118 (1982), 131 (1983), 139 (1984), 165 (1985), 201 (1988), 204 (1988), (1990) [PL]

Phys. Lett.: Cond. Matter (1979-90) [CS]

Phys. Reports (1979-91) [H], 80 (1981), 129 (1985), 142-143 (1986), (1989) [PL]

Phys. Rev. A 13 (1976), 21 (1980), 23-24 (1981) [PL], 25-42 (1982-90) [CS], 31-33 (1985-6), 35 (1987), 37-38 (1988), $41-42$ (1990) [PL]

Phys. Rev. B 16-17 (1977-8), 19 (1979), 21-28 (1980-3) [PL], 25-42 (1982-90) [CS], 28-33 (1983-6), 36-38 (1987-8), 39-42 (1989-90) [PL]

Phys. Rev, C 11 (1975), 24-26 (1981-2), 28-29 (1984), 31-34 (1985-6), 41 (1990) [PL], 31-42 (1985-90) [CS]

Phys. Rev. D 23-26 (1981-2) [PL], (1983-90) [CS], 31-34 (1985-6), 37-38 (1988) [PL]

Phys. Rev. Lett. (1984-90) [CS], 60 (1988),

64-65 (1990) [PL]

Phys. Today (1985-91) [H], (1984-90) [PL]

Plasma Chem. \& Plasma Proc. (1988-91) [CS]

Plasma Phys. Index (1977) [CS]

Powder Difraction (1990) [PL]

Proc. Japan Academy B (1986) [CS]

Proc. Royal Soc. of London, 375-380 (1981), (1982-90), 403-408 (1986) [PL]

Prog. Crystal Growth \& Characterization 5 (1982) [CS]

Prog. Mater. Sci. 27 (1982) [CS]

Prog. Optics, 19-22 (1981-5), 25-26 (1988-9) [PL]

Prog. Surf. Sci. 9 (1979), 12 (1982) [CS], 12-20 (1982-90) [PL]

Prog. Theo. Phys. (1982-90) [PL]

Prog. Theo. Phys. Supp. 90 (1987) [PL]

Quaterly J. Royal Astronom. Soc. 18, 22, 26 (1985) [CS]

Quarterly J. Royal Meteorological Soc. 112 (1986) [CS]

Rev. Math. Phys. 1-2 (1989-90) [CS]

Rev. Modern Phys. (1978), 53 (1981), (1983-87) [PL], (1985-90) [CS]

Rev. Physique App. (1974) [R], (1983-90) [PL]

Rev. Sci. Inst. (1981-90) [CS, PL], (1989-91) [H]

Rev. Solid State Phys. 1-2 (1987-90) [CS]

Rivista Nuovo Cim. 5-9 (1981-6), 11 (1988) [PL]

Sci. Abst. Ser. A.: Phys. Abs. 88-90 (1985-7) [PL]

Sky \& Telescope 58 (1979) [CS]

Solid State Comm. 39-42 (1981-2), 47 (1982) [PL

Solid State Electronics (1979-90) [CS]

Solid State Phys. 21 (1968), 36-37 (1981-83), 39-42 (1985-9) [PL]

Superlattice \& Microstruct. 5 (1989-90) [PL] Surf. Sci. (1990-1) [H], 175-176 (1986) [PL]

The Messenger (1976), 29 (1982), 36 (1984) [CS]

Theo. Chimica Acta (1983-91) [H], 77, 78 [PL]

Thin Solid Films 108 (1983) [CS], 131 (1985) [PL]

Zeit. f. Kristallographie 162-192 (1983-90) [CS], (1990) [PL]

Zeit. f. Naturforschung 38-39 (1982),

45A (1990) [PL]

Zeit. f. Physik A (1980-90) [CS], (1982-90) [PL], (1984) [R]

Zeit. f. Physik B (1981-90) [CS], (1984) [R], (1986-90) [PL]

Zeit. f. Physik C 9 (1981), (1983-90) [PL], (1984) [R]

Zeit. f. Physik D 23 (1976), 49 (1982-3), 53 (1983), (1984) [R], 65 (1986), (1990) [PL] Zeit. f. Physik. Chemie 128 (1981) [PL] 